\title{
Extremal Trajectories for Bounded Velocity Mobile Robots
}

\author{
Devin J. Balkcom and Matthew T. Mason
}

\begin{abstract}
Previous work $[3,6,9,8,7,1]$ has presented the time optimal trajectories for three classes of non-holonomic mobile robots: steered cars that can only go forwards, steered cars that go forwards or backwards, and differential drives. Each of the vehicles is modelled as a rigid body in the plane with velocity and angular velocity controls. The systems are differentiated only by the bounds on the controls, but the optimal trajectories are qualitatively different for each system. We explore this difference by considering the effect that control bounds have on the extremal trajectories of bounded velocity vehicles, where the $e x$ tremal trajectories are defined to be the set of trajectories that satisfy Pontryagin's Maximum Principle, a necessary condition for optimality.
\end{abstract}

\section{Introduction and previous work}

We use the term bounded velocity vehicle to describe any system that can be modelled as a rigid body in the plane, with velocity and angular velocity controls. For these systems, the instantaneous rotation center is constrained to a line rigidly fixed to the robot. Dubins [3], Reeds and Shepp [6], and Balkcom and Mason [1] first derived the time optimal trajectories for various models of steered cars and differential drives. For the steered car, the bounds on velocity and angular velocity are derived from constraints on steering angle and speed; for the diff drive the bounds are derived from a constraint on wheel speed.

The time optimal trajectories for bounded velocity vehicles must satisfy Pontryagin's Maximum Principle. In this paper, we present some geometric interpretations of the Maximum Principle that yield some insight into the structure of the time optimal trajectories of bounded velocity vehicles.

The primary result of the paper is a geometric method for constructing level sets of the Hamiltonian for bounded velocity vehicles. These level sets provide a convenient way to classify the optimal trajectories of many bounded velocity systems. The level sets also provide geometric information about the optimal trajectories even in situations where the differential equations describing the optimal trajectories may not be analytically integrated. An

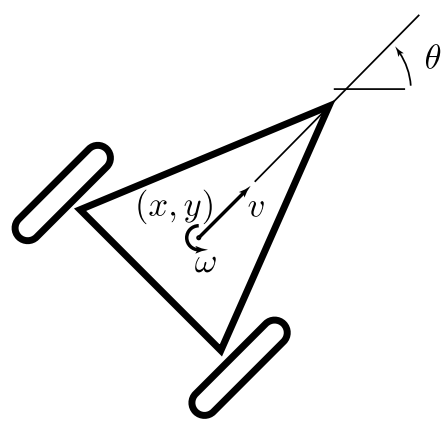

Figure 1: Notation.

additional result of the paper is that the time optimal trajectories for bounded velocity mobile robots may be interpreted as maximizing power along a line of force in the plane.

The results presented depend heavily on work by Dubins [3], Reeds and Shepp [6], Sussman and Tang [9], Souères and Laumond [8], Souères and Boissonat [7], and Balkcom and Mason [1]. Laumond [4] and Balkcom and Mason [2] would be good starting points for those unfamiliar with previous work on time optimal trajectories for steered cars and differential drive vehicles.

\section{System model}

We model all of the vehicles as rigid bodies in the plane. The state of the system is $q(t)=(x(t), y(t), \theta(t))$. We will consider the controls to be $u(t)=(v(t), \omega(t))$, the velocity and angular velocity of the vehicle. (See figure 1.) The system equations are

$$
\dot{q}=v f_{v}+\omega f_{\omega}
$$

where $f_{v}$ and $f_{\omega}$ are the vector fields

$$
f_{v}=\left(\begin{array}{c}
\cos \theta \\
\sin \theta \\
0
\end{array}\right) \quad f_{\omega}=\left(\begin{array}{l}
0 \\
0 \\
1
\end{array}\right)
$$

Vector field $f_{v}$ corresponds to driving forward and and $f_{\omega}$ corresponds to rotation about the reference point. Geometrically, these equations express the constraint that the robot cannot move sideways. 


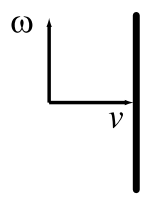

Dubins

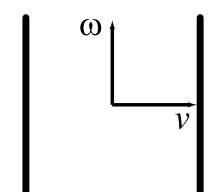

Reeds and Shepp

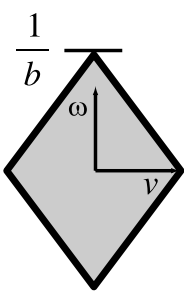

Diff Drive
Figure 2: Control regions for steered cars and differential drives.

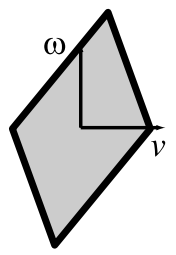

Diff drive with mismatched motors

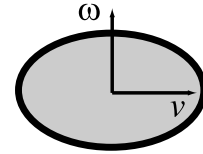

Bounds on effector speed or kinetic energy
Figure 3: Other control bounds.

The set of admissable controls determines the optimal trajectories for a bounded velocity mobile robot. Admissible controls are bounded Lebesgue measurable functions from time interval $[0, T]$ to $\mathbf{R}^{2}$. We assume that there exists some region $U$ such that

$$
(v(t), \omega(t)) \subset U, \text { for all } t \in[0, T]
$$

Figure 2 shows the control regions for the Dubins car, the Reeds and Shepp car, and the differential drive. Figures 3 and 4 show control regions for some systems for which the time optimal trajectories are not known. In the definitions that follow, we assume units have been chosen appropriately, and the reference frame has been affixed to an appropriate place on the robot.

Dubins The car can only drive at one speed, and the steering angle is bounded.

$$
U=\{(v, \omega): v=1,|\omega| \leq 1\}
$$

Reeds and Shepp This car is similar, but we permit the velocity to be positive or negative.

$$
U=\{(v, \omega):|v|=1,|\omega| \leq 1\}
$$

Differential Drive If $b$ is the distance from the center of the axle to either wheel, and we bound the wheel angular velocities, it turns out that

$$
U=\left\{(v, \omega):|v| \leq 1,|\omega| \leq \frac{1-|v|}{b}\right\}
$$

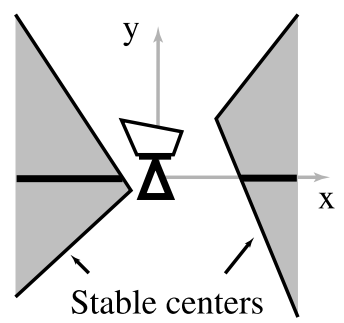

of rotation

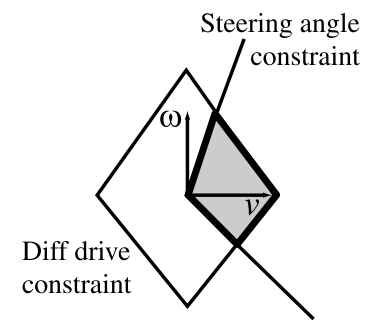

Control Bounds
Figure 4: Quasistatically stable rotation centers and control bounds for a differential drive pushing a block.

In this case, $U$ is a diamond. If the maximum speeds of the wheels are different, then the shape of this diamond may be warped, as shown in figure 3 .

Fast stable pushing by a mobile robot There is a polygon of quasistatically stable rotation centers for a polygonal object being pushed by a polygonal robot. (See Lynch and Mason [5], for example.) We may combine this constraint with the bounded velocity constraint to find $U$. Figure 4 shows the construction of a control region for a differential drive pushing a block.

Bounded control effort Constraints on maximum kinetic energy or on the maximum speed of a camera or effector attached to the robot might give bounds of the form

$$
U=\{(v, \omega): \exists s \text { s.t. } v \leq \cos (s) / \alpha, \omega \leq \sin (s)\}
$$

where $\alpha$ is a positive constant relating the maximum angular rate and the maximum forwards or backwards speed. $U$ is an ellipse, as shown in figure 3 .

\section{Pontryagin's Maximum Principle. Extremal controls.}

Time optimal trajectories have been shown to exist for the Reeds and Shepp car. The other control regions described above are convex, and thus the conditions of Sussmann and Tang's Theorem 6 in [9] may be easily verified to prove existence of optimal trajectories.

We now summarize the results of applying Pontryagin's Maximum Principle to vehicles satisfying system equation 1. For details, the reader is referred to Balkcom and Mason [2].

1. The Hamiltonian for the system is

$$
H(q, u)=v\left(c_{1} \cos \theta+c_{2} \sin \theta\right)+\omega\left(c_{1} y-c_{2} x+c_{3}\right)
$$

where $c_{1}, c_{2}$, and $c_{3}$ are arbitrary constants satisfying $c_{1}^{2}+c_{2}^{2}+c_{3}^{2}>0$. 
2. We define $\lambda_{0}(t)$ to be the negative of the minimum attained for the Hamiltonian. $\lambda_{0}$ is constant in time and non-negative.

3. The control $u(t)$ minimizes the Hamiltonian at almost every $t$.

We say that a trajectory is extremal (satisfies the Maximum Principle) if there exist constants $c_{1}, c_{2}$, and $c_{3}$, such that $c_{1}^{2}+c_{2}^{2}+c_{3}^{2}>0$, the control $u(t)$ minimizes the Hamiltonian at almost every $t$, and $\lambda_{0} \geq 0$. All time optimal trajectories are extremal, but not all extremal trajectories are time optimal. For a differential drive, a spin in place through an angle of $2 \pi$ is extremal, but not time optimal!

If $c_{1}=c_{2}=0$, then equation 8 reduces to

$$
H=c_{3} \omega
$$

Since $c_{3}$ must be non-zero, $\omega(t)$ must be either maximized or minimized at almost every $t$. With the exception of the Reeds and Shepp car, there is a unique control minimizing $\omega$ and a unique control maximizing $\omega$ for each of the example systems. So optimal trajectories corresponding to the case $c_{1}=c_{2}=0$ have constant controls.

If $c_{1}^{2}+c_{2}^{2}>0$, we may assume without loss of generality that $c_{1}^{2}+c_{2}^{2}=1$. (Notice that equation 8 can be scaled by arbitrary postive constants without changing the set of controls satisfying the minimization condition.) If we define

$$
\begin{aligned}
\eta(x, y) & =c_{1} y-c_{2} x+c_{3} \\
\beta(\theta) & =\theta-\operatorname{atan}(-c 2, c 1)
\end{aligned}
$$

then equation 8 can be written

$$
-H=v \cos \beta+\omega \eta
$$

\section{Geometry of the Hamiltonian}

There is a geometric interpretation of $\eta$ and $\beta$. There is a line of points satisfying $\eta(x, y)=0$. The position of the line is determined by the arbitrary constants $c_{1}, c_{2}$, and $c_{3}$. We will call this line the $\eta$-line. If $c_{1}^{2}+c_{2}^{2}=1$, then $\eta(x, y)$ is the signed distance of the reference point of the robot from the $\eta$-line. If we associate a direction with the $\eta$-line consistent with this signed distance, $\beta(\theta)$ is the heading of the robot relative to the $\eta$-line.

It is interesting that the right hand side of equation 12 is a dot product between a generalized velocity and vector dependent on the location of the robot relative to some line. Consider a line of force coincident with the $\eta$-line, but with opposite direction. Assume a force of 1 is applied against the robot, pushing on the robot in the direction opposite the $\eta$-line. With a suitable choice of units, the time derivative of the work done by the robot against this force is

$$
v \cos \beta+\omega \eta
$$

This expression and the right hand side of equation 12 are the same. So informally, the Maximum Principle tells us that the time optimal trajectories for bounded velocity vehicles maximize power along a line of force in the plane; the $\eta$-line is this line of force.

\section{Level sets of the Hamiltonian}

The right hand side of equation 12 is a dot product between the control $u$ and another vector. Define

$$
d=(\cos \beta, \eta)
$$

We call $d$ the characteristic vector. From the Maximum Principle,

$$
\begin{aligned}
\lambda_{0} & =\max (-H(t)) \\
\langle u, d\rangle & =\lambda_{0}
\end{aligned}
$$

That is, the control $u$ must minimize the dot product, and the dot product is constant.

Equations 15 and 16 lead to a useful geometric construction. We first consider the differential drive. Choose a small but nonzero value for $\lambda_{0}\left(0<\lambda_{0}<1\right)$, and consider the point $u_{1}=(1,0)$ on the control boundary, corresponding to the robot driving in a straight line. (See figure 5). There is a line of values for the characteristic vector that make a constant dot product with $u_{1}$, labelled "line 1" in figure 5. Similarly, consider the other three vertices of the control boundary and construct three corresponding lines. If $d$ falls along one of the lines, the dot product in 16 will be equal to $\lambda_{0}$ for at least one of the controls $u_{1}, u_{2}, u_{3}$, or $u_{4}$.

The Maximum Principle gives an additional constraint, described by equation 15 . The fact that the control must be maximizing restricts the possible values of the characteristic vector to the rectangle formed by the four lines, shown in figure 5. No new points are added to or removed from the rectangle when we consider the remaining admissable controls.

If we consider the Hamiltonian as a function of the characteristic vector

$$
H(d)=-\langle u(d), d\rangle
$$

then the constructed rectangle is a level set of the Hamiltonian:

$$
d \in H^{-1}\left(-\lambda_{0}\right)
$$

We may find other level sets of the Hamiltonian by scaling $\lambda_{0}$. Geometrically, increasing $\lambda_{0}$ uniformly scales the 


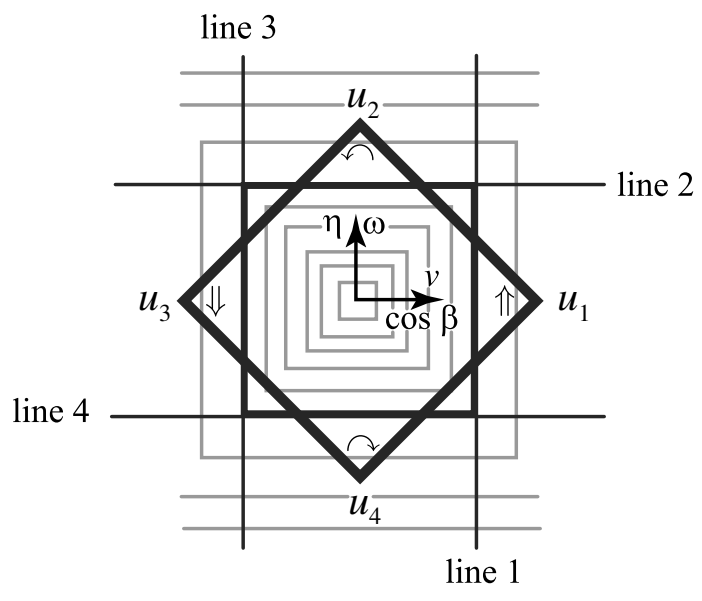

Figure 5: Geometric construction of level sets of the Hamiltonian for the differential drive.

constructed rectangle. However, there is one additional constraint: the first element of the characteristic vector $d$ is $\cos \beta \leq 1$. If $\lambda_{0}$ is large, then parts of the constructed rectangle must be clipped to satisfy this constraint. Figure 5 shows some level sets of the Hamiltonian for the differential drive in gray.

The procedure carried out for the differential drive can be applied to the Dubins car, the Reeds and Shepp car, and some variations. For control regions $U$ with polygonal boundaries, we observe that vertices of the boundary map to edges of level sets of the Hamiltonian, and edges of the boundary map to vertices of level sets.

In order to satisfy the Maximum Principle, the characteristic vector $d$ must fall on a single level set of the Hamiltonian. Furthermore, the characteristic vector is a continuous function of time. If the level set of the Hamiltonian is comprised of disjoint sections ( $\lambda_{0}$ large), then the characteristic vector will be restricted to one of the sections.

Since the level sets of the Hamiltonian are qualitatively different for large and small $\lambda_{0}$, we expect the extremal trajectories to be qualitatively different for large and small $\lambda_{0}$. In fact, the value of $\lambda_{0}$ provides a convenient way to distinguish classes of trajectories for bounded velocity vehicles.

As an example, we construct the level sets of the Hamiltonian for the steered car. For small $\lambda_{0}$, the level sets of the Hamiltonian are diamonds in the control region. For large $\lambda_{0}$, we clip the diamonds to satisfy the constraint that $|\cos \beta| \leq 1$. Figure 6 shows the result. Once the level sets are constructed, we may classify the trajectories by the value of $\lambda_{0}$ :

$\lambda_{0}>1$ : The robot is far from the $\eta$-line, and the maximizing control will either always maximize or minimize
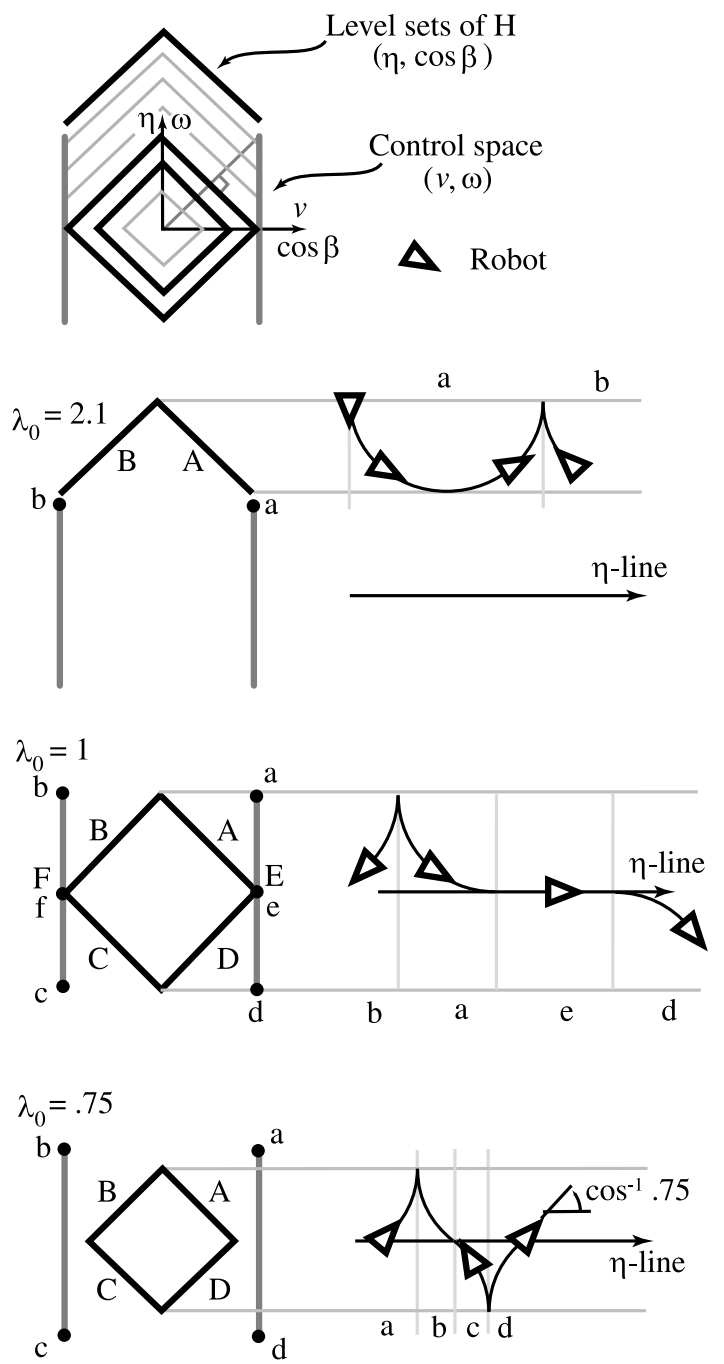

Figure 6: Reeds and Shepp car: level sets of the Hamiltonian and extremals.

$\omega$. The robot follows successive half-circles, switching driving direction but maintaining a constant angular velocity. The second row of figure 6 shows an example.

$\lambda_{0}=1$ : The robot is always either following a $\pi / 2$ arc of a circle with an endpoint on the $\eta$-line, or driving in a straight line along the $\eta$-line. See the third row of figure 6.

$\lambda_{0}<1$ : The robot is near the $\eta$-line, and makes a sharp angle with the $\eta$-line. The extremal trajectory resembles a parallel parking maneuver. See the fourth row of figure 6.

The extremals for differential drives and steered cars are already well understood. However, the level sets of the Hamiltonian can also quickly give some geometric information and allow the extremals to be constructed for other 


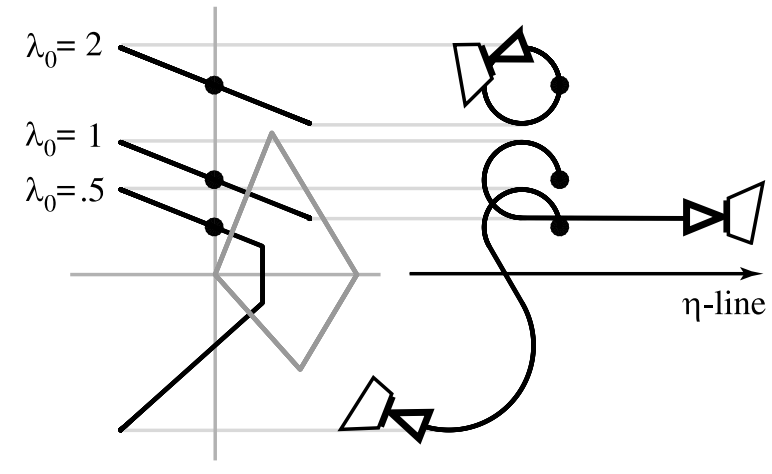

Figure 7: Some level sets of the Hamiltonian and corresponding extremals for a differential drive pushing a block.

systems. Figure 7 shows the level sets of the Hamiltonian for a differential drive pushing a block. The extremals fall into three classes: extremals for which $\lambda_{0}$ is small, singular extremals, and extremals for which $\lambda_{0}$ large. Figure 7 shows some geometrically contructed extremals for each class.

\section{Strictly convex smooth boundary}

The examples considered so far assumed a polygonal boundary of $U$. We now turn to the case where the boundary is smooth and strictly convex. We parameterize the boundary of $U$ by a function $\gamma$ :

$$
\gamma(s)=\left(\begin{array}{c}
v(s) \\
\omega(s)
\end{array}\right)
$$

We will find the level sets of the Hamiltonian by constructing a family of curves. $D_{\lambda_{0}}$ will be a curve containing the level set of the Hamiltonian, for each value of $\lambda_{0}$. We will decribe $D_{\lambda_{0}}$ by a parameterization $\left(d_{1}(s), d_{2}(s)\right)$. From equation 16,

$$
\langle\gamma(s), d(s)\rangle=v(s) d_{1}(s)+\omega(s) d_{2}(s)=\lambda_{0}
$$

Since the boundary is smooth and strictly convex, the maximization condition (equation 15) becomes

$$
\langle\dot{\gamma}(s), d(s)\rangle=\dot{v}(s) d_{1}(s)+\dot{\omega}(s) d_{2}(s)=0
$$

Combining equations 20 and 21 and dropping the $s$ for notational convenience,

$$
\begin{aligned}
d_{1}(s) & =\frac{\lambda_{0} \dot{\omega}}{v \dot{\omega}-\dot{v} \omega} \\
d_{2}(s) & =-\frac{\lambda_{0} \dot{v}}{v \dot{\omega}-\dot{v} \omega}
\end{aligned}
$$

These equations are only defined if the denominator is non-zero. It is interesting to note that the denominator is

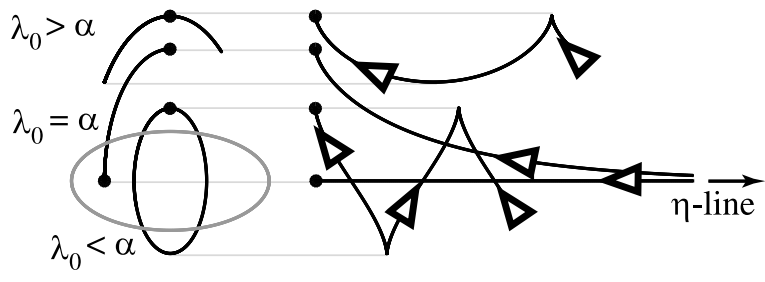

Figure 8: Some level sets of the Hamiltonian and corresponding extremals for a vehicle with a control region bounded by an ellipse.

the Wronskian of $v(s)$ and $\omega(s)$. The Wronskian is only zero over some interval if the two functions are linearly dependent over the interval. Therefore, there is a large class of control regions for which equations 22 and 23 hold almost everywhere.

As an example, we may use equations 22 and 23 to construct the level sets of the Hamiltonian for the control region with elliptical boundary described by equation 7 . The control boundary can be parameterized by

$$
\gamma(s)=\left(\begin{array}{c}
v(s) \\
\omega(s)
\end{array}\right)=\left(\begin{array}{c}
\cos (s) / \alpha \\
\sin (s)
\end{array}\right)
$$

for $\alpha>0$ and $s \in[0,2 \pi)$.

$$
\dot{v}(s)=\frac{-\sin (s)}{\alpha} \quad \dot{\omega}(s)=\cos (s)
$$

Substituting into equations 22 and 23:

$$
\begin{aligned}
& d_{1}(s)=\alpha \lambda_{0} \cos (s) \\
& d_{2}(s)=\lambda_{0} \sin (s)
\end{aligned}
$$

So in fact, the level sets of the Hamiltonian must be ellipses, or subsections of ellipses. Although it seems likely that the extremals for this system can only be described by differential equations that cannot be integrated analytically, the level sets still give useful geometric information. Figure 8 shows the constructed level sets and four numerically generated extremals. We may again classify the extremals by the value of $\lambda_{0}$ :

$\lambda_{0}<\alpha$ : The level set is an ellipse, and $|\cos \beta|<1$. Therefore $\beta \in(0, \pi)$ or $\theta \in(\pi, 2 \pi)$ over the entire trajectory. The control smoothly varies as $(\cos \beta, \eta)$ moves around the ellipse, in either a clockwise or counterclockwise direction. $v$ changes sign when $\beta=\pi / 2$ or $\beta=-\pi / 2$, and $\omega$ changes sign when the robot crosses the $\eta$-line.

$\lambda_{0}=\alpha$ : The level set is an ellipse. If $\beta=0$ or $\beta=\pi$, then the robot will drive either forwards or backwards 
along the $\eta$-line. Otherwise, the robot will follow a trajectory asymptotically approaching the $\eta$-line.

$\lambda_{0}>\alpha$ : The characteristic vector $d$ takes values from a section of the ellipse, either above or below the horizontal axis. Assume the robot starts above the $\eta$-line. Then the controls smoothly vary, but $\omega$ is always positive. If the robot starts below the $\eta$-line, $\omega$ is always negative. Robots following trajectories of this class are are always turning to the right or to the left. If $\lambda_{0}$ is very large, then the control will have large values for $\omega$ and small values for $|v|$. If the $\eta$-line is at infinity $\left(c_{1}=c_{2}=0\right)$, the robot will spin in place.

\section{Other results}

The level sets of the Hamiltonian allow the classification of extremals and the identification of critical values of $\lambda_{0}$. In fact, the level sets of the Hamiltonian can provide a wealth of other geometric information about extremal trajectories. In this section, we briefly summarize some uses of the level sets of the Hamiltonian.

Construction of extremals If the boundary of the control region is polygonal, we may use level sets of the Hamiltonian to construct extremal trajectories. For each edge of the level set there is a corresponding constant control which will cause the robot to follow a line segment, an arc of a circle, or to spin in place. From the length of the edge we may determine the duration for which the control may be executed.

If the boundary of the control region is smooth, we are unlikely to be able to explicitly integrate the differential equations describing the trajectory. However, the constraint that the characteristic vector must lie on a single level set over an extremal can be used to either reduce the number of variables or to guide the numerical integration.

Bounding tubes The level sets of the Hamiltonian may also permit useful geometric observations even if explicit integration is not possible. For example, we may calculate an upper bound on the size of a corridor or tube in the plane that contains all optimal trajectories between some start and goal configuration. If there are no obstacles in the tube, the time optimal trajectory exists and is collision free.

Locating the $\eta$-line Finally, this paper describes the extremal trajectories relative to some line in the plane, the $\eta$-line. However, what we really want is the set of time optimal trajectories between every pair of start and goal configurations. One approach to this problem is to derive the location of the $\eta$-line as a function of the start and goal configurations. The level sets can be used to provide a constraint on the location of the $\eta$-line, based on the observation that the start and the goal must fall on the same contiguous section of a level set.

\section{Conclusion}

The optimal trajectories for mobile robots with velocity and angular velocity controls can be described as maximizing power along a line of force. The power over any trajectory must be constant. Finally, the level sets of the Hamiltonian provide additional useful geometric information, even if the extremal trajectories may not be explicitly constructed.

We would like to thank Yoshihiko Nakamura, Daniel Nikovski, Al Rizzi, and Illah Nourbakhsh for their helpful comments and criticism. We would also like to thank Jean Paul Laumond for his guidance on optimal control for mobile robots. This research was supported by the National Science Foundation under grant IIS-9900322, and by a DOE Computational Science Graduate Fellowship.

\section{References}

[1] D. J. Balkcom and M. T. Mason. Time optimal trajectories for bounded velocity differential drive robots. In IEEE International Conference on Robotics and Automation, 2000.

[2] D. J. Balkcom and M. T. Mason. Time optimal trajectories for differential drive vehicles. International Journal of Robotics Research, to appear.

[3] L. E. Dubins. On curves of minimal length with a constraint on average curvature and with prescribed initial and terminal positions and tangents. American Journal of Mathematics, 79:497-516, 1957.

[4] J. P. Laumond. Nonholonomic motion planning for mobile robots. Technical report, LAAS, 1998.

[5] K. M. Lynch and M. T. Mason. Stable pushing: Mechanics, controllability, and planning. International Journal of Robotics Research, 15(6):533-556, Dec. 1996.

[6] J. A. Reeds and L. A. Shepp. Optimal paths for a car that goes both forwards and backwards. Pacific Journal of Mathematics, 145(2):367-393, 1990.

[7] P. Souères and J.-D. Boissonnat. Optimal trajectories for nonholonomic mobile robots. In J.-P. Laumond, editor, Robot Motion Planning and Control, pages 93-170. Springer, 1998.

[8] P. Souères and J.-P. Laumond. Shortest paths synthesis for a car-like robot. IEEE Transactions on Automatic Control, 41(5):672-688, May 1996.

[9] H. Sussmann and G. Tang. Shortest paths for the reedsshepp car: a worked out example of the use of geometric techniques in nonlinear optimal control. SYCON 9110, Department of Mathematics, Rutgers University, New Brunswick, NJ 08903, 1991. 